\title{
Deciphering the Molecular Role of Non-coding RNA in Malignant Cardiac Disease
}

\author{
Ishita Sharma $^{1}$ (D), Tapan Behl 1,* (D), Aayush Sehgal 1 (D), Monika Sachdeva ${ }^{2}$ (D), Sukhbir Singh 1 (D), \\ Neelam Sharma 1 (D), Simona Bungau 3 (D) \\ 1 Chitkara College of Pharmacy, Chitkara University, Punjab, India \\ 2 Fatima College of Heath Sciences, Al Ain, UAE \\ 3 Department of Pharmacy, Faculty of Medicine and Pharmacy, University of Oradea, Romania \\ * Correspondence: tapanbehl31@gmail.com;
}

Scopus Author ID 56560241200

Received: 20.11.2020; Revised: 14.12.2020; Accepted: 15.12.2020; Published: 17.12.2020

\begin{abstract}
Non-coding RNA (NcRNA) is a functional RNA which contributes to the gene regulation at both transcriptional and post-transcriptional level. NcRNA lack in the encoding of proteins, but this does not counteract the generic functioning of the cells. It is a group of RNA with versatile functioning. They are classified into small nuclear RNA, tRNA, rRNA, and microRNA. The cumulative functioning of ncRNA includes slicing as well as the management of chromosomes. The long ncRNA plays a vital role in malignant cardiovascular disease such as atherosclerosis, myocardial infarction, and injury. They act by different pathways and undergo different mechanisms that include binding to CBX, activating mesoderm posterior-1, modulating MKX25, and targeting bone morphogenetic proteins. They have an intricate role in cardiovascular disorders as some of the long ncRNAs contribute to the inhibition of plague formation in the heart, thus reducing the risk for atherosclerosis and thus retarding the development of heart failure. Whereby microRNA acts as a prognostic biomarker in the early detection of heart injury. MicroRNA has even been identified for the inhibition of angiogenesis, and Piwiinteracting RNA is important in genetic diversity. Thus, ncRNA is one of the factors in the leading cause of malignant cardiac disorder and is responsible for cardiac remodelling andheart failure.
\end{abstract}

Keywords: non-coding RNA; atherosclerosis; myocardial infarction; heart failure; microRNA.

(C) 2020 by the authors. This article is an open-access article distributed under the terms and conditions of the Creative Commons Attribution (CC BY) license (https://creativecommons.org/licenses/by/4.0/).

\section{Introduction}

Non-coding ribonucleic acid (ncRNA) is a functional RNA transcribed from deoxyribonucleic acid (DNA). It does not encode a protein but performs all the functions and even contains different information. Failure to encode a protein does not address the fact that it does not perform any purpose or contain any data, but it does contain certain data. It is also acclaimed that most of the information in mammals is executed through proteins [1]. Despite the fact that they do not encode a protein, recently, a study reported that most of the genomes of complex organisms as well as of mammals are transcribed by ncRNA. In contrast, their functions fulfill the generic functions of cells and help regulate the gene expression at both post-transcriptional and transcriptional levels. Henceforth, few examples of RNA's and their functions include small nuclear RNA (snRNA), which is involved in splicing; transfer-RNA (tRNA) and ribosomal-RNA (rRNA) are involved in the translation of mRNA [2]. Cumulatively it was also concluded that there is a minimum number of ncRNA that is found in prokaryotes. The ones that are present mainly function in the regulation of mRNA translation 
process. However, ncRNA does not dictate the genomic output in prokaryotes. There are few infrastructural ncRNA's that have well-recognized functions. rRNAs, tRNAs, snRNAs, small nucleolar RNA (snoRNAs) are the common infrastructural ncRNA. It is also being known that some of the RNA's are being involved in the regulatory process. snRNA is involved in the splicing, but apart from this, it also regulates the transcription elicited by RNA polymerase- 2 . Moreover, the ncRNA is also involved in the biological processes, in the maintenance of chromosomes, and its segregation $[3,4]$. Apart from this, a few small regulatory ncRNAs were also discovered in plants and animals. These small regulatory ncRNA are divided into two broad categories: miRNA and snoRNA [5]. The small nucleolar RNA is only restricted to rRNA, which is widely acclaimed in the modification for ribosomal biogenesis, but now, apart from modification, targeting of various RNAs, i.e., mRNA and snRNA can also be made. miRNA is derived from both non-coding transcripts and protein-coding. There are few biological roles of ncRNA being outlined and encompasses splicing of RNA, editing of RNA, control of chromosome dynamics, and gene regulation in eukaryotes. Apart from the above, transcription is also regulated by ncRNA, whereas RNA polymerase- 2 is also regulated by ncRNA. The cumulative effect of ncRNA is seen in its response to stress. NcRNA may even act as scaffolding for the complex macromolecular assembly. An example of RNA includes rRNA present in the ribosome [6].

Malignant cardiac diseases are the heart's complication, which encompasses heart failure, cardiac tamponade, and superior vena cava syndrome. Cardiac myxoma is the preoperative diagnosis of malignant heart disease. It is a benign tumor of the heart that is mostly found in the left atrium. Metastasis is most prevalent in malignant cardiac disorders and includes the development of a cardiac neoplasm. Malignant tumors comprise $10 \%$ of primary cardiac tumors that are easily eradicated by surgery [7].

According to the world health organization, these diseases are classified as undifferentiated pleomorphic sarcomas, fibrosarcomas, synovial sarcomas, and carcinogen sarcoma. Carcinogen sarcoma covers $36 \%$ of the frequency for cardiac sarcomas and is effective in the right atrium and pericardium [7]. The pericardium is the most dominant site of this tumor. The most common symptom related to carcinogen sarcoma is chest pain. While during the pericardial biopsies, it is difficult to diagnose the sarcoma, and henceforth, it is mostly mistaken by mesothelioma. The undifferentiated pleomorphic tumors or sarcoma are mainly found in the left atrium and are derived from the endocardium cells. Due to its position in the left atrium, there signs and symptoms are mainly seen in pulmonary vein obstruction, pulmonary hypertension. The cumulative effect of all the above present will then even affect the lungs. Another type of malignant heart disease that occurs on the left side of the heart is osteosarcoma. Fibro-sarcomas, this term is mainly defined to be used for myofibroblast sarcomas of spindle cells (an elongated cell that indicates the presence of a2 sarcoma). Leiomyosarcoma constitutes $6 \%$ of cardiac sarcomas. These usually affect the left atrium, and their action is also seen in the pulmonary veins.

Shortness of breath (dyspnea) is the main clinical feature of leiomyosarcoma [8]. A myxoid feature that is the presence of incomplete peripheral ossification or chondroid matrix, is also another feature associated with this sarcoma. Rhabdomyosarcoma constitutes up to 5\% of cardiac sarcomas [9]. This type of sarcoma does not affect the left atrium but affects the valves of the heart. Lymphoma that constitutes over $20 \%$ of people with non-Hodgkin lymphoma, is evidence that they are involved in the cardiac function at the autopsy level. Usual symptoms seen in such types of lymphomas are edema, arrhythmia, and dyspnea. Their lesions 
are found in the left atrium, pericardium, right ventricle, and right atrium. These types of sarcomas are mostly seen in patients with acquired immunodeficiency syndrome, organ transplant, in patients with a heart transplant.

\section{Heart and Non-Coding RNA}

\subsection{Long non-coding RNAs.}

Nanotechnology Human genome transcribes only $2 \%$ of protein. These ncRNA are classified in accordance with the length of their respective transcripts. The small ncRNA are the ones that are shorter than 200 nucleotides, while above this is the long coding RNAs. Long ncRNAs (lncRNA) execute an imperative function in the formation of tissues in the human body $[9,10]$. These RNAs help in maintaining the morphology and differentiation of the cell. By transcribing at spatial points, long coding RNAs may even contribute to molecular signaling. These mainly act as links between coding and non-coding by the formation of RNADNA complex [11]. The localization of RNA on a cellular level is important for determining the functional properties. When it comes to lncRNA location, they are found in the cytoplasm, and some even reside in the nucleus. The ones found in the nucleus are used in the regulation of genes by forming the RNA-DNA complex [11]. At the same time, the ones located in the cytoplasm regulate the modification of the protein. These RNAs are also linked to ribosome that is used in the development of peptides [12]. The lncRNAs are also found in gene regulation in the cardiovascular system (CVS) that plays an essential responsibility in the heart's functioning and development [13]. The functions that are affected by the lncRNAs are the development of the heart wall, depolarization of cardiac cells, and cardiac morphology. The IncRNAs are seen to potentiate the maintenance of CVS [14]. With this, there are lncRNAs that act as potential biomarkers of the heart. There are many lncRNA that possess specific expressions related to the heart. With major roles to play, lncRNA acts as a potential mediator for maintaining cardiovascular diseases, and they also act as potential biomarkers (Table 1) [14-20].

Table 1. Clinically relevant lncRNAs in the cardiovascular system.

\begin{tabular}{l|l|l|l} 
Transcript & Host Genes & Mechanisms & Diseases \\
\hline Anril & $\begin{array}{l}\text { CDKN2B- } \\
\text { AS1 }\end{array}$ & $\begin{array}{l}\text { Binds to CBX7 that further recruits PRC-1, } \\
\text { PRC-2 lead to P-15, P-16 transcription } \\
\text { repression }\end{array}$ & Risk aspect for coronary artery ailment \\
\hline BVHT & BVHT & $\begin{array}{l}\text { Activation of mesoderm posterior-1 that } \\
\text { interacts with PRC-2 }\end{array}$ & Cardiomyocyte differentiation impairment \\
\hline FENDRR & FENDRR & $\begin{array}{l}\text { Binds to PRC-2 complex and TrxG to } \\
\text { change chromatin }\end{array}$ & Cardiac hypoplasia \\
\hline NOVLNC6 & Intergenic & Modulation of MKX25 & Down regulation in cardiomyopathy \\
\hline SENCR & SENCR & Inhibit the migration of smooth muscle cell & Down regulation in myocardial infarction \\
\hline H19 & H19 & Acts by targeting VDAC1 & $\begin{array}{l}\text { Regulates cardiomyocyte apoptosis in diabetic } \\
\text { cardiomyopathy }\end{array}$ \\
\hline LIPCAR & JA760602 & - & Mostly elevated in patients with cardiac heart failure \\
\hline GAS5 & GAS5 & $\begin{array}{l}\text { It interacts with miR-290 that induce the } \\
\text { downstream gene expression }\end{array}$ & $\begin{array}{l}\text { This transcript induces hypertension that induces } \\
\text { micro-vascular disorder }\end{array}$ \\
\hline CHRF & DCC & It targets miR-489 & Regulates the cardiac hypertrophy \\
\hline PANCR & PITX2 & miR-143 and miR-501 & Affects in arterial fibrillation \\
\hline HOTAIR & HOTAIR & Targets NOX2 & Up-regulation is seen in ischemic heart failure
\end{tabular}

BVHT: Braveheart; CBX7: Chromobox hamolog-7; PRC-1: protein-coding; VDAC1: voltage-dependent anionselective channel; GAS5: growth arrest-specific; PITX2: pair like homeodomain transcription factor-2: miR-143: Micro RNA.

\subsection{MicroRNAs (miRNA).}


Small ncRNAs are also known as miRNAs, single-stranded molecules with less than 22 nucleotides in their strands. They primarily function as regulators of post-transcriptional for the regulation of genes [20]. These are the functional unit of RNA, i.e., RNA-induced silencing complex. This slicing complex leads to the degradation of mRNA by binding to its target mRNA. By inducing a new layer, these micro RNAs can even be sequester by long coding RNAs. Apart from being an integral part of cardiovascular, miRNAs even play an important in the maturation of smooth muscles, endothelial dysfunction, and regulation of that are involved in cardiogenesis. If any dysregulation is found in miRNAs, it may affect the heart and cause pathological effects like; atherosclerosis, cardiomyopathy, and even heart failure. The first miRNA was developed in 2008, that is miR-21, and it is used in cardiac dysfunction. Several pathogenic conditions include cardiomyopathy, myocardial fibrosis, atherosclerosis, and heart failure caused by dysregulation of miRNA (Table 2) [21].

Table 2. Clinically relevant microRNA in the cardiovascular system.

\begin{tabular}{l|l|l} 
Transcript & Mechanism & Disease \\
\hline miR-133 & De-repression of IRX5 & $\begin{array}{l}\text { Regulating the balance between proliferation and } \\
\text { differentiation in cardiogenesis }\end{array}$ \\
\hline miR-208a & Regulates the balance between myosin chain & Its inhibition is protective in heart failure \\
\hline miR-17 & Targets BMP, TBX1 genes & Regulates the genes of cardiac progenitor \\
\hline miR-195 & Regulates CHEK1 & Associated with hypoplasia of right ventricle \\
\hline miR-25 & Regulates cardiac contractility by SERCA 2a & Heart failure mitigation \\
\hline miR-590 & - & Stimulation of cardiac regeneration \\
\hline miR-21 & Regulation of the activity of ER-MAP kinase & It ameliorates cardiac dysfunction. \\
miR: microRNA; BMP: Bone morphogenetic proteins.
\end{tabular}

\subsection{Circular RNAs ( $\operatorname{circRNA).}$}

CircRNA is a class of ncRNA that has been developed by changing the sequence of RNA. It is one of the emerging classes found in a variety of species like fishes, fungi, insects, and even mammals. It has been found that it performs multiple functions in different and numerous biological processes. Splicing, protein binding, translation, transcription is a few of the functions being performed by circular [22]. Their main function is the production of protein in a cap-independent manner. These are produced using a different type of machinery, i.e., translational machinery. Apart from the production of proteins, they even perform a critical task in the pathogenesis and encompass the production of various diseases that includes cancer and CVD [23]. CircRNA has been identified during the pathological condition of the heart. Different gene expressions play a major role in cardiovascular biology, and they include titin (TTN) and ryanodine receptor-2 (RYR2) genes. This RNA has a life medium of around 2.5 times higher than their respective linear counterparts [24].

Table 3. Clinically relevant circular RNAs in the cardiovascular system.

\begin{tabular}{l|l|l|l} 
Transcript & Host gene & Mechanism & Disease \\
\hline HRCR & PWWP2A & miRNA acts as a sponge for miRNA-223 & Inhibition of heart failure \\
\hline MICRA & ZNF609 & - & $\begin{array}{l}\text { Downregulation is seen in case of } \\
\text { heart failure }\end{array}$ \\
\hline circANRIL & CDKN2B-AS1 & It binds to PES1 & It protects against atherosclerosis \\
\hline CircFOXO3 & FOXO3 & $\begin{array}{l}\text { It helps in retaining the aging factors-E2F1, } \\
\text { ID1 in the cytoplasm }\end{array}$ & $\begin{array}{l}\text { Upregulation is seen in myocardial } \\
\text { senescence }\end{array}$ \\
\hline cZNF292 & ZNF292 & - & Promotion of angiogenesis
\end{tabular}

HRCR: hypersensitivity response secretion protein; ZNF609: zinc finger protein-609; PES1: pescadillo ribosomal biogenesis; E2F-1: E2F transcriptional factor-1; ID1: inhibition of DNA binding-1.

These are also highly stable RNA, and apart from this, they are even there in the plasma as well as circulated well in the blood. Apart from this, they even have the potential to act as 
therapeutics [22,23]. During cardiac development and pathological conditions, there are different transcripts of circRNA that conduct a fundamental role in cardiovascular biology. Table 3 summarizes the role of circRNA in the development of cardiovascular diseases.

\section{Nature of NcRNAs}

NcRNAs have the most conserved nature; especially the miRNAs are the most conserved ones. The genomes related to ultra-conserved ones are mainly present in intergenic regions as well as in introns. The conservation of ncRNA is possessed at different levels of like-sequence level, expression level, structural level, and positional conservation.

\subsection{Sequence level.}

The nature of sequence level conservation has been used precisely in the detection of homologous transcripts. Different studies were being performed on different species so as to see the conserved nature of IncRNAs [25]. The studies that were performed included Nescules et al.'s study conducted on 11 vertebrates that further showed RNA sequencing of different tissues. Another study that was conducted for this was the PLAR study, which was conducted on 17 vertebrates [26]. The result came out to be that RNA sequencing in multiple organisms was seen. Apart from conducting the studies on vertebrates, it was even conducted on insect species. In total, 7 insect species were taken that showed non-long coding RNA in insect species [26].

However, in many cases, this transcriptase profile and transcribed sequence may not provide a complete perspective. Moreover, in a few organisms, there is no similarity in the sequence between the different organisms. The similarity is only seen near the 5' end and even at the lncRNA promoter sequence. The promoter region of this RNA is conserved, just like the ones present in protein-coding genes. Apart from insects and avian species, there are numerous IncRNAs present in humans with a sequence, including HOTAIR, CARMEN, CHAST, and GAS5 [26,27].

\subsection{Synteny level.}

The ncRNA controls the expression of gene proteins by means of both Trans-and-cis mechanisms. At this point, when it comes to terms of their sequence and transcription, most of them experience fast evolution. Despite this evolution, its relationship with the neighboring genes remains the same and conserved. The vast majority of genes preserved are only at the RNA promoter region [28-30]. The lncRNAs present in cardiovascular biology and are even found in conserved locations are PVT1 and FENDRR. These are the ones that do not show any type of conservation identified in the sequence.

The position of lncRNA performs an incredibly critical task in its mechanism. As already studied, cis is one of the mechanisms of lncRNAs, so its importance is mainly seen in this mechanism. The lncRNAs act as antisense for the genes that encode the proteins. This has been a great influence on proteins [28]. They work on every aspect, especially by interacting with proteins of their genes, RNA, and even DNA. The retention in the evolution of lncRNA is seen at that time when it overlaps the protein-coding genes [29]. This retention is seen because they represent tissue specificity. This shows their function in the neighboring genes and even in the chromatin modulation. 


\subsection{Structure level.}

The miRNAs are one of the types of ncRNA that are known to form secondary structures and also play a significant role in the interaction of RNA with different biomolecules. These lncRNA are also capable enough to form a stable structure just like other RNAs form. The absence of sequence level will not cause any effect in the selection of genes at the structure level [31]. The Stem region of miRNA and telomerase RNA are two such examples that even maintain the structural integrity despite the absence of sequence. Any random RNA can even form a sequence with a stable structure, but it doesn't need to function accordingly at the sequence level [32]. It is not sure to date about the conservation of the structure of RNA. Many different hypotheses are being made for it. A few cardiovascular RNA have some sought of structural conservation; they are HOTAIR and GAS5. Most of the studies based on the secondary structure of genome RNA showed that a certain degree of sequence conservation is required as a tool for sequence alignment [32,33].

\section{Explicating the NcRNA in Cardiovascular Diseases}

Earlier being considered junk, they never played an important role in CVD. However, now after so many evolutionary techniques, ncRNA is being measured as a new achievement in cardiovascular diseases. As already studied, there are two types of lncRNA, i.e., miRNA and ncRNA. Their main role is seen in regulating myocardial infarction, atherosclerosis, insulin resistance, and diabetic cardiomyopathy. RNAs play a vital role in the treatment as well as the diagnosis of CVD. Out of them, especially the miRNA plays a significant function in the early detection of any heart disease.

\subsection{Mechanism of miRNA.}

Nanotechnology miRNA possesses a length of 18-25 nucleotides, and it also regulates the expression of genes by binding with mRNA. RNAs are abundantly present in almost all the tissues of the body and are more than 2500 in numbers present in a single genome of humans [34]. miRNAs are mostly transcribing from the introns, with the help of enzyme RNA polymerase 2 or sometimes even from RNA polymerase-3 depending upon the function [35]. RNA polymerase- 3 is transcribed into a primary miRNA molecule. These primary miRNAs further also get converted into pre-miRNA by undergoing a maturation process by a specific enzyme, i.e., DROSHA, that makes miRNA cleaves it into pre-miRNA [35,36]. Further, with the help of exportin-5 these are forwarded into the cytoplasm for their further functioning. As soon as they come out of the nucleus, they again endure cleavage. This time, the cleavage is due to the enzyme Rnase-3, and thereby they are cleaved into mature and double-stranded miRNA [34,35]. The mature miRNA possesses an activated strand.

The activated strand firstly binds to the RISC, i.e., RNA induced silencing complex, and then will impede in the Argounate- 2 and trans-activation response RNA binding protein (TRBP) that further leads to the inhibition of target mRNA 3'UTR position by the interaction of base and pairs [37,38]. Presence of binding sequence in mRNA regulates the number of targets it possesses, as mRNA can possess multiple targets. Translational inhibition or degradation of target mRNA depends upon the complementarily of sequence between mRNA and miRNA. A very significant aspect that renders the mRNA and miRNA complex is the nucleotide sequence present in the 5' end of miRNA. Apart from this, miRNA can even be generated from the strands of DNA donated by a suffix; 3-p and 5-p [37]. A passenger strand 
that is also an opposite strand is usually degraded because of its lower steady-state levels, and it is even represented by an asterisk (*). In some cases, it is seen that these passenger strands are also useful in the case of miRNA and even become functional to miRNA that also targets mRNA [20,21]. miRNA loci are present in numerous genomic contexts; while most of them are present in the intronic region, they could also be encoded in exonic regions.

Many different pathways are being followed by miRNAs, like a canonical and noncanonical pathway that leads to the maturation $[25,26]$. In the non-canonical pathway, short introns work by the splicing method. Splicing is usually done of DROSHA, i.e., known as mitron pathway [27-29]. Several miRNAs come under this class, including small nucleor RNAs, tRNAs, and short hairpin RNA [37]. So, there are different pathways for the miRNA biogenesis that shows the complexity of the processing of RNA. For slicing a target transcript, there are mirtrons and simtrons capable of doing this activity. The ones targeted and interacted by this process are argonaute proteins. Regardless of all this, many mechanisms work for the maturation of miRNA to function actively in the cardiovascular systems (Figure 1).

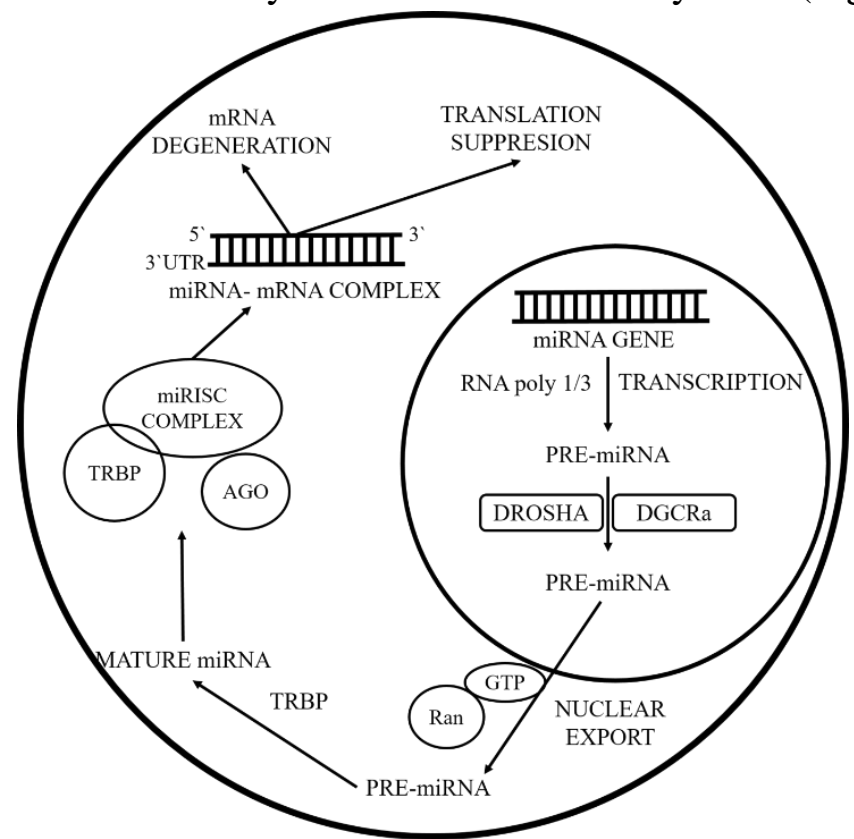

Figure 1. Biogenesis of miRNA and their mode of succession of transcripts. TRBP: Transactivation response element RNA binding protein; Ago: argonaute family of proteins; DROSHA: initiates the step of miRNA in the nucleus.

\subsection{Mechanism of IncRNA.}

These represent a vast variety of ncRNA with reverence to their diverse mechanisms, have different characteristics, and have different localization even. As the name suggests, these are longer, i.e., they have more than 200 nucleotides and even regulate the expression of genes at both levels, i.e., at transcriptional and non-transcriptional levels [39,40]. This RNA is mainly synthesized by RNA polymerase 2 . They are synthesized along with the 5'capping and polyend chain. When it comes to protein-coding DNA, their functioning is done with cis-and-trans elements for DNA sequences coding; thereby, they also act as epigenetic mediators [37]. These are made the same as transcripts of mRNA also to perform synthesis accordingly. However, apart from this, they lack in not having an open reading frame (ORF). By adding one more layer for the transcriptional regulation, this IncRNA can even be regulated by miRNAs; this makes up adding one more layer of transcriptional regulation [41]. LncRNA can thereby also act as miRNA sponges as it consists of complementary sequences to miRNA. LncRNA is 
classified in accordance with their length of the genome, their respective position and mechanism of action, protein-coding elements, and even their association with the DNA. These are classified in accordance with their genomic function and their loci [36]. Sence RNA is the first type synthesized from the exons of protein-coding genes using the same gene promoter [36]. Antisense RNA is synthesized from the protein-coding region but from their opposite strand and is known as aberrant transcripts $[42,43]$. As the name already suggests, intronic RNA is introduced from introns of the same as that of a protein-coding gene. Enhancer IncRNA, these are synthesized from activators of protein-coding genes [44]. CircRNA forms a circular loop by enclosing 3'-5'end. This circular loop is again formed from the splicing of proteins $[36,40]$. Bidirectional transcriptions are transcribed from the opposite direction but the same promoter as coding genes [36]. Intergenic RNAs are the ones that transcribe independently and are even encoded between the protein-coding genes [36,40]. As studied, there are numerous numbers of lncRNA present, but when coming on to their functioning, it is not adequately identified. Their main role is seen in the organ's development, and they are even studied in CVD.

\subsection{PIWI-Interacting RNA (piRNA).}

This is probably the first class that is different from lncRNA and miRNA. They are usually found in the genomes cluster and usually range in between 25-30 nucleotides length $[36,45,46]$. This interacts with p-element induces wimpy testis, i.e., PIWI, which belongs to the family of argonaute; they by this means lead to the silencing of a complementary sequence by the formation of ribonucleoprotein complex that then recognized the complementary sequence $[47,48]$. These piRNA PIWI complex are associated with targeting transposable elements in the somatic cells and even regulates the transcription [36,49]. The mechanism is not as such known for this. However, they regulate the genome sequence, and this regulation is made canonically that involves the cleavage of endonucleolytic through a special splicing complex, i.e., piRISC complex. In the murine genome, there are 50,000 piRNA sequences. These execute an imperative task in the regulation of genetic diseases. They even play an important role in genetic diversity [50,51] (Figure 2).

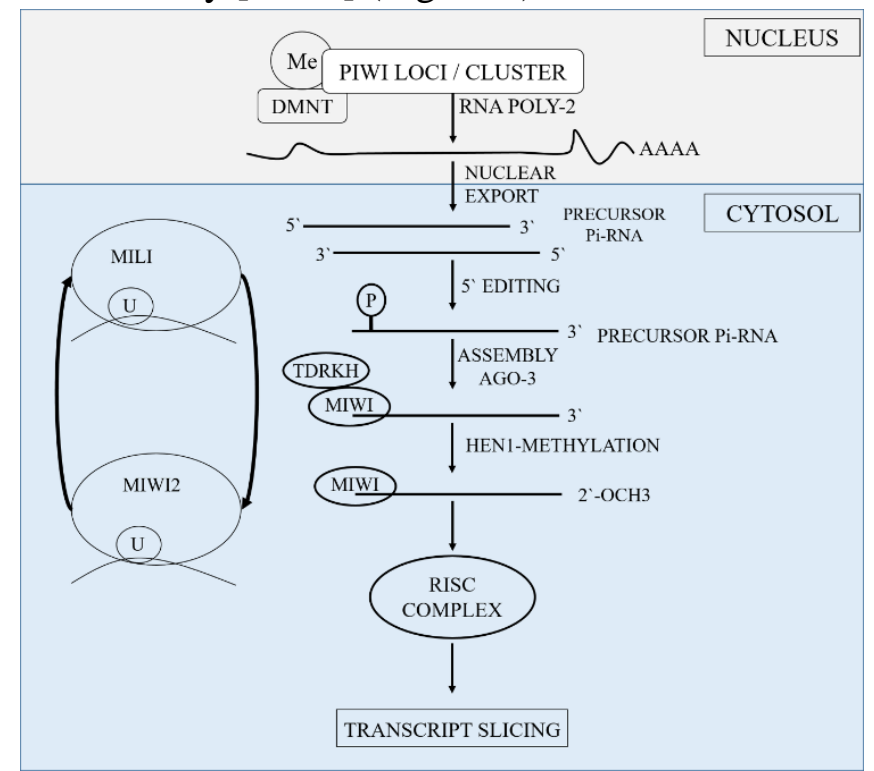

Figure 2. Synthesis of Pi-RNA. piRNA, piwi protein interaction RNA; DNMT, DNA methyltransferase; HEN1, HUA ENHANCER 1; Miwi, mouse homolog of PIWI; AGO3, argonaute 3. 


\section{MicroRNAs in Myocardial Infarction}

Ischemia and infarction are the main reason for the apoptotic death of cardiomyocytes. These deaths are being regulated by miRNA, which is a subtype of lncRNA. The most potent ones that regulate it are miR-304b, miR-34a, miR-21, miR-15b, and miR-497 [37]. Almost all these regulate them negatively and target anti-apoptotic genes, i.e., BCL-2. MiRNA usually regulates the injury of myocardial infarction and re-perfusion, and it is also inversely interrelated to the BCL-2 expression. These are inversely related to this expression in cardiomyocytes [43]. A study conducted by Pan et al. showed that levels of miR-1 and LNA anti-miR-1 exhibit alleviated levels in the injury [52]. Whereas the study even demonstrated that inhibition of miR-1 would reduce cardiac cell death, which could be inhibited by regulation of HSP60, protein kinase $\mathrm{C}$. This injury causes further induction of miR-1 with downregulation of BCL-2 [53]. While if we see in the case of cardiomyocyte that is induced due to stress, in that miR-1 and miR-133 possess elevated levels. MiR-1 seems to have a pro-apoptotic role, while miR-133 has an anti-apoptotic role [54]. There is an elevation in the levels of miR-1 in the post-myocardial infarction; these suppress the anti-apoptotic genes. The anti-apoptotic genes are; Hsp-70, Bcl-2, IGF-1, and Hsp-60 [55,56]. The patients who died of myocardial infarction showed decreased levels of miR-133. These reduced levels were seen in the infracted parts of the heart [37]. A different miRNA, i.e., miR-320, is regulated after the heart's injury in the murine hearts. The ones with over-expression of this miR-320 showed the death of cardiomyocytes. Reduction of the miR-320 with antagomir-320 showed a reduction in cardiomyocyte death. HSP20 was the one that helped in targeting miRNA; it also acts as cardioprotection against the injury [57,58].

Another miRNA, miRNA-494, has also gathered attention. It is down-regulated in the failing hearts and even protects one against myocardial injury. It works by reducing the apoptosis and the size of infarct [59]. Similarly, in the case of over-expression, it will lead to the inhibition of caspase- 3 that will eventually decrease the probability of cardiomyocyte death in I/R injury. Mir-21 is known to carry out a critical function in reducing ischemia/re-perfusion (I/R) injury via triggering the signaling pathway of pro-survival in cardiomyocytes and by targeting numerous pro-apoptotic genes that include Fas ligand, PTEN, and programmed cell death-4 [37,60,61]. PTEN also executes an imperative role in the signaling pathway's activation, and its inhibition decreases the infarct size. After an acute myocardial infarction, miR-21 induces cardioprotective effects in the heart. It even improves left ventricular and also reduces the size of the infarct $[62,63]$. Stimulation of miR-21 through hydrogen sulfide is the best therapy for the treatment of infusion/infarction injury. MiR-21 is induced in the early stage only so that it acts as a protection in infarction injury. While miR-21 is induced in the last stage of injury, it can cause cardiac remodeling and fibrosis [37,59].

Another miR, miR-17-92, consists of a family of almost 7 sub members, and they are miR-20a, miR-19b-1, miR-18a, and miR-17. MiR-17-92 helps in controlling the cardiomyocytic death and is even great in the proliferation [64,65]. AntagiomiR-92a is the one that enhances angiogenesis and even helps in reducing apoptosis [37,43]. MiR-126 is the most protective miRNA. It promotes the formation of new blood vessels in the heart. These blood vessels are formed by regulating different mediums that are VEGF, vascular cell adhesion, angiopoietin-1 [37,54]. MiR-126 was even observed in the endothelium bodies, leading to apoptosis in the injury by regulating CXCL12 production. In the development of cardiovascular, ephrin-A3 plays a vital role in it. In hypoxia, ephrin-A3 is inhibited by mir- 
210 , leading to angiogenesis in response to an ischemic condition $[37,59,66]$. Overload of calcium in mitochondria also contributes to the injury of infarction/ischemia. By targeting at sodium/calcium exchanger, miR-214 acts as a cardio-protector for the injury to act against the increased calcium level in the body [67]. This RNA acts as a protection for the heart against the overproduction of calcium, as overproduction leads to cardiomyocyte death $[37,68]$.

\section{MicroRNAs and Atherosclerosis}

Atherosclerosis is a progressive disorder of coronary arteries that is usually caused by the formation of plague in the arteries and even by the deposition of fats, accompanied by inflammation in the internal wall of blood vessels [69]. This accumulation will lead to narrowing the vessels that will eventually cause complications related to myocardial infarction. Several miRNAs act as potential regulators in the process involved in the development of atherosclerosis, for instance, liquid uptake, efflux, and cellular adhesion [44]. Many hepatic enriched miRNA have been identified that have a major impact on lipid homeostasis. Hepaticmir-122 is a hepatic enriched miRNA that regulates the synthesis of cholesterol and fatty acids in the body $[37,70,71]$. Another miRNA that has been implicated in cholesterol efflux includes miR-26, miR-144, mir-758, miR-33, and miR-106. Among these, mir-33 is the most broadly investigated. It also represses the numerous genes concerned with cellular cholesterol trafficking. Mir-33a/b has entrenched within the introns of SREBP (sterol regulatory elementbinding proteins); these are the regulators of lipogenic genes and cholesterolemia genes [72,73]. The SGERP is the most important regulator for the cholesterol genes [74]. Its transcription will inhibit the gene miR-33 that will further lead to inhibition of efflux of cholesterol. This inhibition process will occur by acting on ATP transporters that include ABCA1 (ATP binding cassette transporter A1) and ABGE1 [37,75]. The anti-miR-33 effects will automatically increase HDL levels by the efflux of cholesterol by two different genes: ABCA1 and ABCG1. Apart from miR-33, miR-26 and miR-302a are used in the transporting and efflux of cholesterol by acting on ABAC-1 [76,77].

Atherosclerosis is even promoted by inflammatory activation of endothelial cell (EC) by leukocyte recruitment. The molecules used in the enrollment of leukocytes are intracellular adhesion molecules, E-selectins, and vascular adhesion molecules that eventually lead to the formation of plague [78]. When it comes to EC's functioning in human beings, ICAM and Eselection target pro-inflammatory cytokines; TNF-alpha is induced at the genes of miR-17-3p and miR-31, which eventually leads to the regulation of adhesion of neutrophils in EC [37]. MiR-181b leads to the regulation of Nf-kappa B mediated activation of EC, atherosclerosis, and vascular inflammation using subjugation of importin- $\alpha 3$, a protein that is necessary for the nuclear translocation of NF-kB [37,78]. MiR-146 upholds vascular homeostasis amid repression of the different pro-inflammatory signaling pathways that include, NF- $\kappa$ B pathway in addition to the MAP kinase pathway and downstream early growth response (EGR) transcription factors throughout the regulation of IL- $1 \beta$ signaling pathway adaptor proteins (i.e., TRAF6, IRAK1/2) [79]. Mir-146 helps modulate the pro-inflammatory pathways by a different medium, like via targeting the human antigen R. This RNA binding protein promotes the activation of endothelial by endothelial nitric oxide pathway [37]. Regulation of vascular inflammation is mainly associated with miRNA-126, limiting the adherence of leukocytes by suppressing VCAM-1. Thereby it's over-expression will show beneficial results by decreasing the accumulation in the blood vessels in case of atherosclerosis by decreasing the levels of 
TNF-alpha and 1L-6. These accumulations are reduced by inhibition of the MAPK pathway (JNK and p38) [37,80].

Elevation in the level of miR-365 was seen when ox-LDL was treated with HUVECs; apart from elevation, downregulation of its target Bcl-2was also there [37]. However, miR-365 inhibition attenuated ox-LDL-induced the apoptosis of EC by restoring the phrase of Bcl-2. While in the case of atherosclerosis and the formation of plague, miR-365 was found to be down-regulated. The levels of $1 \mathrm{~L}-16$ that are the direct targets of miR-365, in both monocytes and plague interrelated using the expression level of miR-365; this makes for its role in the pathogenesis of atherosclerosis [81,82]. It also reduces the propagation of the vascular smooth muscle cells by targeting the CD-1 i.e., cell cycling D1. Another miR-92 is the one that is seen found in EC due to oxidative stress in the body and even contributes to atherosclerosis development. This targets three of the untranslated region of different mRNA that includes klf2 and klf4, i.e., krupple like factors, and even by sirtuin-1 [37].

MiR-27a leads to the macrophage foam cell formation that initiates atherosclerosis, which is activated by CD68, CD14, and CD206 and with the secretion of IL-10. The levels of IL-10 are elevated by the activation of a pathway that is the ERK signaling pathway [83]. MiR155 is even there to promote atherosclerosis in the heart by repressing bcl-6 expression (B-cell leukemia) and NF- $\kappa \mathrm{B}$ pathway, i.e., a transcription factor [37,83]. Atherosclerotic plague can be reduced by the total delivery of antagomiR155; it also diminishes the lipid loading in macrophages. MiR-155 over-expression identified that HBP1, i.e., HMG box transcription protein-1 acts as a new target of miR-155. By the process of direct suppression of HBP1, foam cell formation can be achieved by producing ROS of the macrophages and by the promotion of liquid uptake [84]. Additionally, miR-155 also straightforwardly inhibits SOCS1 expression and stimulates p-STAT3 and PDCD4, leading to inflammation mediator's production in macrophages to endorse atherosclerotic plaque formation [85].

Up-regulation of miR-217 was revealed to negatively control Silent information regulator 1 (Sirt1) that is present in human atherosclerotic plaques [86]. Inhibition of miR-217 restores the levels of Sirt1 and modulates forkhead box O1 (FoxO1), and enhanced angiogenesis [87]. Sirt1 is an aging master regulator that directly targets miR-217 and declines overage. Mir-34a over-expression will enhance the body's pro-inflammatory factors that further even increase LDL level in the body. Inhibition of the same will lead to a decrease in atherosclerotic lesions and eventually decrease EC apoptosis [37].

MiR-21 has established important attention with respect to its function in CVDs as it was revealed to be up-regulated in atherosclerosis patients. In prior atherosclerosis stages, miR21 exhibits the effect of pro-inflammatory in ECs via commencement of pro-inflammatory protein VCAM1 and MCP-1 (monocyte chemotactic protein-1) [37,88,89]. Nevertheless, in the subsequent stages of the pathological phase, inflammation regulation can be eased by inducing eNOS with enhanced development of atheroprotective NO, which suppresses monocyte activation and adhesion and expression pro-inflammatory cytokines. Many studies have established the controversial function of miR-21 as a pro- or anti-atherogenic miRNA [37,90-92]. Shear stress plays a crucial role in atherosclerosis. It contributes to its severity by increasing the plague formation in the veins [33]. Regulation of shear stress is made by miR663. Analysis of up-regulation of miR-663 is mainly done by HUVECs. Hence all these miRs are sensitive towards shear stress as it contributes to the formation of plague. Apart from shear stress, abnormal plague modeling in the veins also contributes to atherosclerosis. For this, miR29 regulates positively, reducing the chances of plague formation in the veins and vessels. 
Thereby, every miR directly or indirectly functions in the negative and positive regulation of atherosclerosis in the heart [37].

\section{Cardiovascular Diseases and LncRNA}

Conventional lncRNAs help in regulating differentiation, apoptosis, and proliferation of the cell [37]. These are mainly found and expressed in many conditions like cardiovascular disorders, cancer, and even diabetes. LncRNA works very precisely and powerfully in the development of the heart. The first known lncRNA was myosin, a heavy chain RNA transcript that acts in the proliferation of cardiomyocytes [37]. Heavy chain transcripts include; Mhrt that is impeded via Brg1-HDAC-PARP-a chromatin repressor that governs the change of alphaMHC to beta-MHC. Pathological strain further activates Brg1, represses Mhrt, and, therefore, leads to cardiac hypertrophy in adults [37,93]. A considerable function for Braveheart (BVHT) IncRNA in the cardiac lineage is being observed that it directs the mesoderm toward the cardiac fate via a mediator of cardiovascular progenitors named mesoderm posterior-1 (MesP1). Bvht interacts with SUZ12, a core constituent of polycomb repressive complex-2 (PRC2), suggesting epigenetic monitoring of chromatin. Moreover, several significant transcription factors essential for the commitment of cardiac lineages like MesP1, Gata4, Hand1, Hand2, Nkx2.5, and Tbx5 are triggered through Bvht [37].

LNcRNA that is cardiac enriched contributes to the heart's cardiac hypertrophy by blocking the methylation in H3 histone by interacting with PRC2 [37,94]. Chast is another cardiomyocyte that belongs to the lncRNA category, which up-regulates the aortic of stenosis patients. It hampers cardiomyocytes and helps in promoting hypertrophy in the heart. Apart from this, H19 levels were also found to be elevated in the case of atherosclerosis [37,94]. Their over-expression will precisely lead to enhancement of P38 and P65, thereby reducing apoptosis in the HUVEC (human umbilical vein endothelial cells). Autophagy is one of the mechanisms that eradicate proteins and the organelles that are damaged from the system to decrease the cellular burden in the body. AK079427 is the only autophagy of lncRNA that regulates the heart's injury due to myocardial infarction or atherosclerosis by decreasing the miR-188-3p [37,93].

Likewise, cardiac autophagy inhibitory factor (CAIF) suppresses cardiac autophagy and assuage myocardial infarction (MI). CAIF straightforwardly associates with p53 protein and precludes its linking to the promoter region of myocardia, and terminates its transcription [95]. The loss of myocardin, in order, depletes the accretion of LC3-II and ameliorates autophagy. The transcription factor of myocardin in cardiomyocytes is important for the healthy growth of the sarcomere. Myocardin ablation induces cardiomyocyte destruction due to increased apoptosis, which contributes to dilated cardiomyopathy [96]. During I/R injury, the homeostasis of mitochondrial dynamics in the heart is important. Mitochondrial fusion can suppress apoptosis, although an increasing body of evidence suggests that cellular apoptosis in the pathogenesis of many disorders may be triggered by abnormal mitochondrial fission. Prohibitin complexes, PHB1, and PHB2, are present in the inner mitochondrial membrane and play an important role in mitochondrial fusion and fission [97]. PHB1-overexpressing transgenic mice that are subjected to MI showed it reduced mitochondrial fission and lesser myocardial infarct size. On the other hand, Wang et al. demonstrated that over-expression of PHB2 inhibits post-ischemic mitochondrial fission by reducing myocardial apoptosis and MI. They also identified that PHB2 is negatively regulated by miR-539, affecting mitochondrial fission and apoptosis [98]. Intriguingly, the same study revealed that cardiac apoptosis-related 
lncRNA (CARL) acts as a sponge and negatively regulates miR-539 and enhances PHB2 expression to inhibit mitochondrial fission and myocardial apoptosis, consequently attenuating MI [37]. The autophagy-promoting factor will eventually cause suppression of mir-188-3p in ischemic re-perfusion injury. Meg3 in myocardial hypertrophy will cause cardiac remodeling by targeting MMP-2 genes [36,37,51,98-104].

\section{Conclusions}

Conventional ncRNAs are functional RNA that contributes to both transcriptional and non-transcriptional levels and lacks protein-encoding but performs all vital functions. The ncRNA has a direct relationship with the heart. The role of ncRNA in malignant cardiac diseases is intricate as they have a vital role in the inhibition of plague formation in atherosclerosis, cardiac hypertrophy, and hyperplasia as well as a prognostic biomarker. As mentioned, ncRNA has been classified as IncRNA, miRNA, circRNAs, and piwi RNA. The cumulative effect of all the above is seen in cardiac dysfunction, cardiac hypoplasia, cardiac hypertrophy, heart failure inhibition, and inhibiting the formation of plague in atherosclerosis. Non-coding genes function in contrast to fulfill the generic functions of various cells.

LncRNA has been shown to play a vital role in cardiac lineage and act as one of the important regulators of heart functionality. They tend to suppress the risk of heart failure and inhibit plague formation. In patients of heart failure, miRNA tends to act as progestic biomarker in determining infarction and heart failure at possible early stages. CircRNA is involved in the progression of cardiac heart diseases. A prototype of this, i.e., ANRIL, was found to be present in the risk factor for atherosclerosis. While it's another prototype, circANRIL, is a therapeutic agent for treating plague formation during atherosclerosis. In summary, ncRNA act as potential therapeutics; they may elicit a new treatment for various diseases in the future.

\section{Funding}

This review received no external funding.

\section{Acknowledgments}

The authors express gratitude to Chitkara College of Pharmacy, Chitkara University, Punjab, India, for motivational support for this review's compilation.

\section{Conflicts of Interest}

The authors declare no conflict of interest.

\section{References}

1. Hadjicharalambous, M.R.; Lindsay, M.A. Long non-coding RNAs and the innate immune response. Noncoding RNA 2019, 5, https://doi.org/10.3390/ncrna5020034.

2. Wang, J.; Samuels, D.C.; Zhao, S.; Xiang, Y.; Zhao, Y.Y.; Guo, Y. Current research on non-coding ribonucleic acid (RNA). Genes 2017, 8, https://doi.org/10.3390/genes8120366.

3. Mattick, J.S., Makunin, I.V. Small regulatory RNAs in mammals. Hum. Mol. Genet. 2005, 14, 121-132, https://doi.org/10.1093/hmg/ddi101.

4. Prinz, F.; Kapeller, A.; Pichler, M.; Klec, C. The implications of the long non-coding RNA NEAT1 in noncancerous diseases. Int. J Mol. Sci. 2019, 20, https://doi.org/10.3390/ijms20030627.

5. Klec, C.; Prinz, F.; Pichler, M. Involvement of the long non-coding RNA NEAT 1 in carcinogenesis. Mol. Oncol. 2019, 13, 46-60, https://doi.org/10.1002/1878-0261.12404. 
6. Mattick, J.S. Non-coding RNAs: the architects of eukaryotic complexity. EMBO Rep. 2001, 2, 986-991, https://doi.org/10.1093/embo-reports/kve230.

7. Ng, A.K.; Bernardo, M.P.; Weller, E.; Backstrand, K.H.; Silver, B.; Marcus, K.C.; Tarbell, N.J.; Friedberg, J.; Canellos, G.P.; Mauch, P.M. Long-term survival and competing causes of death in patients with earlystage Hodgkin's disease treated at age 50 or younger. J. Clin. Oncol. 2002, 20, 2101-2108, https://doi.org/10.1200/JCO.2002.08.021.

8. Lindsay, M.A.; Griffiths-Jones, S.; Young R.S.; Ponting C.P. Identification and function of long non-coding RNAs. Essays Biochem. 2013, 54, 113-126, https://doi.org/10.1042/bse0540113.

9. Roomi, S.; Sherazi, M.F.; Ulla, W.; Khan, O.A.; Minalyan, A.; Atiq, M.; Thalambedu, N. Rhabdomyosarcoma from uterus to heart. J. Community Hosp. Intern. Med. Perspect. 2020, 10, 245-249, https://doi.org/10.1080/20009666.2020.1760465.

10. Guan, J.; Liu, P.; Wang, A.; Wang, B. Long non-coding RNA ZEB2-AS1 affects cell proliferation and apoptosis via the miR-122-5p/PLK1 axis in acute myeloid leukemia. Int. J. Mol. Med. 2020, 46, 1490-1500, https://doi.org/10.3892/ijmm.2020.4683.

11. Jiang, C.; Ding, N.; Li, J.; Jin, X.; Li, L.; Pan, T.; Huo, C.; Li, Y.; Xu, J.; Li, X. Landscape of the long noncoding RNA transcriptome in human heart. Brief. Bioinformatics 2019, 20, 1812-1825, https://doi.org/10.1093/bib/bby052.

12. Carlevaro-Fita, J.; Rahim, A.; Guigó, R.; Vardy, L.A.; Johnson, R. Cytoplasmic long non-coding RNAs are frequently bound to and degraded at ribosomes in human cells. RNA 2016, 22, 867-882.

13. Archer, K.; Broskova, Z.; Bayoumi, A.S.; Teoh, J.P.; Davila, A.;Tang, Y.; Su, H.; Kim, I.M. Long non-coding RNAs as master regulators in cardiovascular diseases. Int. J Mol. Sci. 2015, 16, 23651-23667, https://doi.org/10.3390/ijms161023651.

14. Babapoor-Farrokhran, S.; Gill, D.; Rasekhi, R.T. The role of long non-coding RNAs in atrial fibrillation. Heart Rhythm 2020, 17, 1043-1049, https://doi.org/10.1016/j.hrthm.2020.01.015.

15. Xue, Z.; Hennelly, S.; Doyle, B.; Gulati, A.A., Novikova, I.V.; Sanbonmatsu, K.Y.; Boyer, L.A. A G-rich motif in the lncRNA Braveheart interacts with a zinc-finger transcription factor to specify the cardiovascular lineage. Mol.Cell. 2016, 64, 37-50, https://doi.org/10.1016/j.molcel.2016.08.010.

16. Grote, P.; Wittler, L.; Hendrix, D.,Koch, F.;Währisch, S.; Beisaw, A.; Macura, K.; Bläss, G.; Kellis, M.; Werber, M.; Herrmann, B.G. The tissue-specific lncRNA Fendrr is an essential regulator of heart and body wall development in the mouse. Dev. Cell. 2013, 24, 206-214, https://doi.org/10.1016/j.devcel.2012.12.012.

17. Ounzain, S., Michelett, R.; Beckmann, T.; Schroen, B.; Alexanian, M.; Pezzuto, I.; Crippa. S.; Nemir, M.,;Sarre, A.; Johnson, R.; Dauvillier J. Genome-wide profiling of the cardiac transcriptome after myocardial infarction identifies novel heart-specific long non-coding RNAs. Eur. Heart J 2015, 36, 353-368, https://doi.org/10.1093/eurheartj/ehu180.

18. Bell, R.D.; Long, X.; Lin, M.; Bergmann, J.H.; Nanda, V.; Cowan, S.L.; Zhou, Q.; Han, Y.; Spector, D.L.; Zheng, D.; Miano, J.M. Identification and initial functional characterization of a human vascular cell-enriched long non-coding RNA. Arterioscler Thromb Vasc Biol. 2014, 34, 1249-1259, https://doi.org/10.1161/ATVBAHA.114.303240.

19. Kumarswamy, R.; Bauters, C.; Volkmann, I.; Maury, F.; Fetisch, J.; Holzmann, A.; Lemesle, G.; de Groote, P.; Pinet, F., Thum, T. Circulating long non-coding RNA, LIPCAR, predicts survival in patients with heart failure. Circ. Res. 2014, 114, 1569-1575, https://doi.org/10.1161/CIRCRESAHA.114.303915.

20. Song, Z.; Gao, R.; Ya, B. Potential roles of microRNA-1 and microRNA-133 in cardiovascular disease. Rev Cardiovasc Med. 2020, 21, 57-64, https://doi.org/10.31083/j.rcm.2020.01.577.

21. Zhao, X.; Wang, Y.; Sun, X. The functions of microRNA-208 in the heart. Diabetes Res. Clin. Pract. 2020, 160, https://doi.org/10.1016/j.diabres.2020.108004.

22. Boeckel, J.N.; Perret, M.F.; Glaser, S.F.; Seeger, T.; Heumüller, A.W.; Chen, W.; John, D.; Kokot, K.E.; Katus, H.A.; Haas, J.; Lackner, M.K. Identification and regulation of the long non-coding RNA Heat2 in heart failure. J. Mol. Cell. Cardiol. 2019, 126, 13-22, https://doi.org/10.1016/j.yjmcc.2018.11.004.

23. Yu, F.;Tie, Y.; Zhang, Y.; Wang, Z.; Yu, L.; Zhong, L.; Zhang, C. Circular RNA expression profiles and bioinformatic analysis in coronary heart disease. Epigenomics 2020, 12, 439-454, https://doi.org/10.2217/epi2019-0369.

24. Kristensen, L,S.; Andersen, M.S.; Stagsted, L.V.; Ebbesen, K.K.; Hansen, T.B.; Kjems J. The biogenesis, biology and characterization of circular RNAs. Nat. Rev. Genet. 2019, 20, 675-691, https://doi.org/10.1038/s41576-019-0158-7.

25. Altenhoff, A.M.; Boeckmann, B.; Capella-Gutierrez, S.;Dalquen, D.A.; DeLuca, T.; Forslund, K.; HuertaCepas, J.; Linard, B.; Pereira, C.; Pryszcz, L.P.; Schreiber, F. Standardized benchmarking in the quest for orthologs. Nat. Methods 2016, 13, 425-430, https://doi.org/10.1038/nmeth.3830.

26. Necsulea, A.; Soumillon, M.; Warnefors, M.; Liechti, A.; Daish, T.; Zeller, U.; Baker, J.C.; Grützner, F.; Kaessmann, H. The evolution of lncRNA repertoires and expression patterns in tetrapods. Nature 2014, 505, 635-640, https://doi.org/10.1038/nature12943.

27. Rusinova, I.; Forster, S.; Yu, S.; Kannan, A.; Masse, M.; Cumming, H.; Chapman, R.; Hertzog, P.J. Interferome v2. 0: an updated database of annotated interferon-regulated genes. Nucleic Acids Res. 2012, 41, 1040-1046, https://doi.org/10.1093/nar/gks1215. 
28. Villegas, V.E.; Zaphiropoulos, P.G. Neighboring gene regulation by antisense long non-coding RNAs. Int.J Mol. Sci. 2015, 16, 3251-3266, https://doi.org/10.3390/ijms16023251.

29. Amaral, P.P.; Leonardi, T.; Han, N.; Viré, E.; Gascoigne, D.K.; Arias-Carrasco, R.; Büscher, M.; Pandolfini, L.; Zhang, A.; Pluchino, S.; Maracaja-Coutinho, V. Genomic positional conservation identifies topological anchor point RNAs linked to developmental loci. Genome Bio. 2018, 19, https://doi.org/10.1186/s13059-0181405-5.

30. Ning, Q.; Li. Y.; Wang, Z.; Zhou, S.; Sun, H.; Yu, G. The evolution and expression pattern of human overlapping lncRNA and protein-coding gene pairs. Sci. Rep. 2017, 7, https://doi.org/10.1038/srep42775.

31. Smith, M. A.; Gesell, T.; Stadler, P.F.; Mattick, J.S. Widespread purifying selection on RNA structure in mammals. Nucleic Acids Res. 2013, 41, 8220-8236, https://doi.org/10.1093/nar/gkt596.

32. Rivas, E.; Clements, J.; Eddy, S.R. Lack of evidence for conserved secondary structure in long non-coding RNAs. Nat. Methods. 2017, 14.

33. Kino, T.; Hurt, D.E.; Ichijo, T.; Nader, N.; Chrousos, G.P. Noncoding RNA gas5 is a growth arrest-and starvation-associated repressor of the glucocorticoid receptor. Sci. Signal. 2010, 3, https://doi.org/10.1126/scisignal.2000568.

34. Mayama, T.; Marr, A.K.; Kino, T. Differential expression of glucocorticoid receptor non-coding RNA repressor Gas5 in autoimmune and inflammatory diseases. Horm Metab Res. 2016, 48, 550-557, https://doi.org/10.1055/s-0042-106898.

35. Landthaler, M.; Yalcin, A.; Tuschl, T. The human DiGeorge syndrome critical region gene 8 and Its D. melanogaster homolog are required for miRNA biogenesis. Curr. Biol 2004, 14, 2162-2167, https://doi.org/10.1016/j.cub.2004.11.001.

36. Kishore, R.; Garikipati, V.N.; Gonzalez C. Role of circular RNAs in cardiovascular disease. J. Cardiovasc. Pharmacol. 2020, 6, 128-137, https://doi.org/10.1097/FJC.0000000000000841.

37. Das, A.; Samidurai, A.; Salloum, F.N. Deciphering non-coding RNAs in cardiovascular health and disease. Front. Cardiovasc. Med. 2018, 5, https://doi.org/10.3389/fcvm.2018.00073.

38. Kalayinia, S., Arjmand, F.; Maleki, M.; Malakootian, M; Singh, C.P. MicroRNAs: Roles in cardiovascular development and disease. Cardiovasc Patho. 2020, 50, https://doi.org/10.1016/j.carpath.2020.107296.

39. Zhu, L.; Li, N.; Sun, L.; Zheng, D.; Shao G. Non-coding RNAs: The key detectors and regulators in cardiovascular disease. Genomics 2020, https://doi.org/10.1016/j.ygeno.2020.10.024.

40. Marchese, F.P.; Raimondi, I.; Huarte, M. The multidimensional mechanisms of long non-coding RNA function. Genome Biol. 2017, 18, https://doi.org/10.1186/s13059-017-1348-2.

41. Gehani, S.S.; Agrawal-Singh, S.; Dietrich, N.; Christophersen, N.S.; Helin, K.; Hansen K. Polycomb group protein displacement and gene activation through MSK-dependent H3K27me3S28 phosphorylation. Mol. Cell 2010, 39, 886-900, https://doi.org/10.1016/j.molcel.2010.08.020.

42. Kim, T.K.; Hemberg, M.; Gray, J.M.; Costa, A.M.; Bear, D.M.; Wu. J.; Harmin, D.A.; Laptewicz, M.; Barbara-Haley, K., Kuersten, S.; Markenscoff-Papadimitriou. E. Widespread transcription at neuronal activity-regulated enhancers. Nature 2010, 465, 182-187, https://doi.org/10.1038/nature09033.

43. Tang, Y.; Zheng, J.; Sun, Y.; Wu, Z.; Liu, Z.; Huang, G. MicroRNA-1 regulates cardiomyocyte apoptosis by targeting Bcl-2. Int. Heart J. 2009, 50, 377-387, https://doi.org/10.1536/ihj.50.377.

44. Moore, K.J.; Rayner, K.J., Suárez, Y.; Fernández-Hernando, C. The role of microRNAs in cholesterol efflux and hepatic lipid metabolism. Annu. Rev. Nutr. 2011, 31, 49-63, https://doi.org/10.1146/annurev-nutr081810-160756.

45. O'Donnell, K.A.; Boeke, J.D. Mighty Piwis defend the germline against genome intruders. Cell. 2007, 129, 37-44, https://doi.org/10.1016/j.cell.2007.03.028.

46. Seto, A.G.; Kingston, R.E.; Lau, N.C. The coming of age for Piwi proteins. Mol. cell. 2007, 26, 603-609, https://doi.org/10.1016/j.molcel.2007.05.021.

47. Siomi, M.C.; Sato, K.; Pezi, D.; Aravin, A.A. PIWI-interacting small RNAs: the vanguard of genome defence. Nat. Rev. Mol. Cell Biol. 2011, 12, 246-258, https://doi.org/10.1038/nrm3089.

48. Li, M.; Yang, Y.; Wang, Z.; Zong, T.; Fu, X.; Aung, L.H.; Wang, K.; Wan, J.X.; Yu, T. Piwi-interacting RNAs (piRNAs) as potential biomarkers and therapeutic targets for cardiovascular diseases. Angiogenesis 2020, 4, 1-6, https://doi.org/10.1007/s10456-020-09750-w.

49. Wu, X.; Pan, Y.; Fang, Y.; Zhang, J.; Xie, M.; Yang, F.; Yu, T.; Ma, P.; Li, W.; Shu Y. The biogenesis and functions of piRNAs in human diseases. Mol. Ther. Nucleic Acids 2020, 38, 108-120, https://doi.org/10.1016/j.omtn.2020.05.023.

50. Cordaux, R.;Batzer, M.A. The impact of retrotransposons on human genome evolution. Nat. Rev. Genet. 2009, 10, 691-703, https://doi.org/10.1038/nrg2640.

51. Turkieh, A., Charrier, H.; Dubois-Deruy, E.; Porouchani, S.; Bouvet, M.; Pinet, F. Noncoding RNAs in cardiac autophagy following myocardial infarction. Oxid.Med.Cell. Longev. 2019, 2019, https://doi.org/10.1155/2019/8438650.

52. Pan, Z.; Sun, X.; Ren, J.; Li, X.; Gao, X.; Lu, C.; Zhang, Y.; Sun, H.; Wang, Y.;Wang, H.; Wang, J. miR-1 exacerbates cardiac ischemia-reperfusion injury in mouse models. PloS One 2012, 7, 505-515, https://doi.org/10.1371/journal.pone.0050515. 
53. Kang, B.;Hong, J.; Xiao, J.; Zhu, X.; Ni, X.; Zhang, Y.; He. B.; Wang Z. Involvement of miR-1 in the protective effect of hydrogen sulfide against cardiomyocyte apoptosis induced by ischemia/re-perfusion. Mol. Biol. Rep. 2014, 41, 6845-6853, https://doi.org/10.1007/s11033-014-3570-2.

54. Ye, Y.; Perez-Polo J.R.; Qian, J.; Birnbaum, Y. The role of microRNA in modulating myocardial ischemiareperfusion injury. Physiol. Genomics https://doi.org/10.1152/physiolgenomics.00130.2010.

55. Zhu, H., Fan, G.C. Role of microRNAs in the reperfused myocardium towards post-infarct remodelling. Cardiovasc. Res. 2012, 94, 284-292, https://doi.org/10.1093/cvr/cvr291.

56. Yu , X.Y.; Song, Y.H, Geng Y.J.; Lin Q.X.; Shan, Z.X.; Lin, S.G.; Li, Y. Glucose induces apoptosis of cardiomyocytes via microRNA-1 and IGF-1. Biochem. Biophys. Res. Commun. 2008, 376, 48-52, https://doi.org/10.1016/j.bbrc.2008.09.025.

57. Lorenz, M.W.; Markus, H.S.; Bots, M.L.; Rosvall, M.; Sitzer, M. Prediction of Clinical Cardiovascular Events With Carotid Intima-Media Thickness. Clin. Perspect. Circulation 2007, 115, 459-467, https://doi.org/10.1161/CIRCULATIONAHA.106.628875.

58. Dueñas, A.; Expósito, A; Aranega, A.; Franco, D. The role of non-coding RNA in congenital heart diseases. J. Cardiovas. Dis. 2019, 6, https://doi.org/10.3390/jcdd6020015.

59. Wang, X.; Zhang, X.; Ren, X.P.; Chen, J.; Liu, H.; Yang, J.; Medvedovic, M.; Hu, Z.; Fan, G.C. MicroRNA494 targeting both pro-apoptotic and anti-apoptotic proteins protects against ischemia/reperfusion-induced cardiac injury. Circulation 2010, 122, 1308-1318, https://doi.org/10.1161/CIRCULATIONAHA.110.964684.

60. Cheng, Y.; Liu, X.; Zhang, S.; Lin, Y.; Yang, J.; Zhang, C. MicroRNA-21 protects against the H2O2-induced injury on cardiac myocytes via its target gene PDCD4. J. Mol. Cell. Cardiol. 2009, 1, 5-14, https://doi.org/10.1016/j.yjmcc.2009.01.008.

61. Lv, G.; Shao, S.; Dong, H.; Bian, X.; Yang, X.; Dong, S. MicroRNA-214 protects cardiac myocytes against H2O2-induced injury. J. Cell. Biochem. 2014, 115, 93-101.

62. Sayed, D.; He, M.; Hong, C.; Gao, S.; Rane, S.; Yang, Z.; Abdellatif, M. MicroRNA-21 is a downstream effector of AKT that mediates its anti-apoptotic effects via suppression of Fas ligand. J. Biol. Chem. 2010, 285, 20281-20290, https://doi.org/10.1074/jbc.M110.109207.

63. Keyes, K.T.; Xu, J.; Long, B.; Zhang, C.; Hu, Z.; Ye, Y. Pharmacological inhibition of PTEN limits myocardial infarct size and improves left ventricular function post infarction. Am. J. Physiol. Heart Circ. 2010, 298, 1198-1208, https://doi.org/10.1152/ajpheart.00915.2009.

64. Mogilyansky, E.; Rigoutsos, I. The miR-17/92 cluster: a comprehensive update on its genomics, genetics, functions and increasingly important and numerous roles in health and disease. Cell Death Diff. 2013, 20, 1603-1614, https://doi.org/10.1038/cdd.2013.125.

65. Foshay, K.M.; Gallicano, G.I. miR-17 family miRNAs are expressed during early mammalian development and regulate stem cell differentiation. Dev. Biol. 2009, 326, 431-443, https://doi.org/10.1016/j.ydbio.2008.11.016.

66. Fasanaro, P.; D'Alessandra, Y.; Di Stefano, V.; Melchionna, R.; Romani, S.; Pompilio, G.; Capogrossi, M.C.; Martelli, F. MicroRNA-210 modulates endothelial cell response to hypoxia and inhibits the receptor tyrosine kinase ligand Ephrin-A3. J. BioL. Chem. 2008, 283, 15878-15883, https://doi.org/10.1074/jbc.M800731200.

67. Aurora, A.B.; Mahmoud, A.I.; Luo, X.; Johnson, B.A.; Van Rooij, E.; Matsuzaki, S.; Humphries, K.M.; Hill, J.A.; Bassel-Duby, R.; Sadek, H.A.; Olson, E.N. MicroRNA-214 protects the mouse heart from ischemic injury by controlling $\mathrm{Ca} 2+$ overload and cell death. J. Clin. Inves. 2012, 122, https://doi.org/10.1172/JCI59327.

68. Frey, N.; McKinsey, T.A.; Olson, E.N. Decoding calcium signals involved in cardiac growth and function. Nat. Med. 2000, 6, 1221-1227, https://doi.org/10.1038/81321.

69. Tabas, I.; García-Cardeña, G.; Owens, G.K. Recent insights into the cellular biology of atherosclerosis. $J$. Cell Biol. 2015, 209, 13-22, https://doi.org/10.1083/jcb.201412052.

70. Churov, A.; Summerhill, V.; Grechko, A.; Orekhova, V.; Orekhov, A. MicroRNAs as potential biomarkers in atherosclerosis. Int J. Mol. Sci. 2019, 20, https://doi.org/10.3390/ijms20225547.

71. Elmen, J.; Lindow, M.; Silahtaroglu, A.; Bak, M.; Christensen, M.; Lind-Thomsen, A.; Hedtjärn, M.; Hansen, JB; Hansen, H.F.; Straarup, E.M.; McCullagh, K. Antagonism of microRNA-122 in mice by systemically administered LNA-antimiR leads to up-regulation of a large set of predicted target mRNAs in the liver. Nucleic acids Res. 2008, 36, 1153-1162, https://doi.org/10.1093/nar/gkm1113.

72. Price, N.L.; Rotllan, N.; Canfrán-Duque, A.; Zhang, X.; Pati, P.; Arias, N.; Moen, J.; Mayr, M.; Ford, D.A.; Baldán, Á.; Suárez, Y. Genetic Dissection of the Impact of miR-33a and miR-33b during the Progression of Atherosclerosis. Cell Rep. 2017, 21, 1317-1130, https://doi.org/10.1016/j.celrep.2017.10.023.

73. Canfrán-Duque A., Lin, C.S.; Goedeke, L.; Suárez, Y.; Fernández-Hernando, C. Micro-RNAs and highdensity lipoprotein metabolism. Arterioscler. Thromb. Vasc. Biol. 2016, 36, 1076-1084, https://doi.org/10.1161/ATVBAHA.116.307028.

74. Najafi-Shoushtari, S.H.; Kristo, F.; Li. Y.; Shioda, T.; Cohen, D.E.; Gerszten, R.E.; Näär, A.M. MicroRNA33 and the SREBP host genes cooperate to control cholesterol homeostasis. Science 2010, 328, 1566-1569, https://doi.org/10.1126/science.1189123. 
75. Marquart, T.J.; Allen, R.M.; Ory, D.S.; Baldán, Á. miR-33 links SREBP-2 induction to repression of sterol transporters. PNAS 2010, 107, 12228-12232, https://doi.org/10.1073/pnas.1005191107.

76. Sun, D.; Zhang, J.; Xie, J.; Wei, W.; Chen, M.; Zhao, X. MiR-26 controls LXR-dependent cholesterol efflux by targeting ABCA1 and ARL7. FEBS Lett. 2012, 586, 1472-1479.

77. Meiler, S.; Baumer, Y.; Toulmin, E.; Seng, K.; Boisvert, W.A. MicroRNA 302a is a novel modulator of cholesterol homeostasis and atherosclerosis. Arterioscler. Thromb. Vasc. Biol. 2015, 35, 323-331, https://doi.org/10.1161/ATVBAHA.114.304878.

78. Hajibabaie, F.; Kouhpayeh, S.; Mirian, M.; Rahimmanesh, I.; Boshtam, M.; Sadeghian, L.; Gheibi, A.; Khanahmad, H.; Shariati, L. MicroRNAs as the actors in the atherosclerosis scenario. J. Physiol. Bioch. 2020, 76, 1-2, https://doi.org/10.1007/s13105-019-00710-7.

79. Cheng, H.S.; Sivachandran, N.; Lau, A.; Boudreau, E.; Zhao, J.L.; Baltimore, D.; Delgado-Olguin, P.; Cybulsky, M.I.; Fish, J.E. Micro RNA-146 represses endothelial activation by inhibiting pro-inflammatory pathways. EMBO Mol. Med. 2013, 5, 1017-1034, https://doi.org/10.1002/emmm.201202318.

80. Solly, E.L.; Dimasi, C.G.; Bursill, C.A.; Psaltis, P.J.; Tan, J. MicroRNAs as therapeutic targets and clinical biomarkers in atherosclerosis. J. Clinical Med. 2019, 8, https://doi.org/10.3390/jcm8122199.

81. Qin, B.; Xiao, B.; Liang, D.; Xia, J.; Li, Y.; Yang, H. MicroRNAs expression in ox-LDL treated HUVECs: MiR-365 modulates apoptosis and Bcl-2 expression. Biochem. Biophys. Res. Commun. 2011, 410, 127-133, https://doi.org/10.1016/j.bbrc.2011.05.118.

82. Shoeibi, S. Diagnostic and theranostic microRNAs in the pathogenesis of atherosclerosis. Acta. Physiologica. 2020, 228, https://doi.org/10.1111/apha.13353.

83. Gulei, D.; Raduly, L.; Broseghini, E.; Ferracin, M.; Berindan-Neagoe, I. The extensive role of miR-155 in malignant and non-malignant diseases. Mol. Asp. Med. 2019, 70, 33-56, https://doi.org/10.1016/j.mam.2019.09.004.

84. Tian, F.J.; An, L.N.; Wang, G.K.; Zhu, J.Q. Li, Q.; Zhang, Y.Y.; Zeng, A.; Zou, J.; Zhu, R.F.; Han, X.S.; Shen N. Elevated microRNA-155 promotes foam cell formation by targeting HBP1 in atherogenesis. Cardiovasc. Research. 2014, 103, 100-110, https://doi.org/10.1093/cvr/cvu070.

85. Ye, J.; Guo, R.; Shi Y.; Qi, F.; Guo, C.; Yang, L. miR-155regulatcdindammation response by the S0CS1STAT3-PDCD4 ads in athemgenesis [J/OL]. Medators Infamm. 2016, 2016, https://doi.org/10.1155/2016/8060182.

86. De-Yébenes, V.G; Briones, A.M.; Martos-Folgado, I.; Mur, S.M.' Oller, J.; Bilal, F.; González-Amor, M.; Méndez-Barbero, N.; Silla-Castro, J.C.; Were, F.; Jiménez-Borreguero, L.J. Aging-associated miR-217 aggravates atherosclerosis and promotes cardiovascular dysfunction. Arterioscler. Thromb. Vasc. Biol. 2020, 40, 2408-2424, https://doi.org/10.1161/ATVBAHA.120.314333.

87. Zhang, L.; Chen, J.; He, Q.; Chao, Z.; Li, X.; Chen, M. MicroRNA-217 is involved in the progression of atherosclerosis through regulating inflammatory responses by targeting sirtuin 1. Mol. Med. Rep. 2019, 20, 3182-3190, https://doi.org/10.3892/mmr.2019.10581.

88. Braun, M.; Pietsch, P.; Schrör, K.; Baumann, G.; Felix, S.B. Cellular adhesion molecules on vascular smooth muscle cells. Cardiovas. Res. 1999, 41, 395-401, https://doi.org/10.1016/S0008-6363(98)00302-2.

89. Suárez, Y.; Wang, C.; Manes, T.D.; Pober, J.S. Cutting edge: TNF-induced microRNAs regulate TNFinduced expression of E-selectin and intercellular adhesion molecule-1 on human endothelial cells: feedback control of inflammation. J. Immunol. Res. 2010, 184, 21-25, https://doi.org/10.4049/jimmunol.0902369.

90. Chen, W.J.; Zhang, M.; Zhao, G.J.; Fu, Y.; Zhang, D.W.' Zhu, H.B.; Tang. C.K. MicroRNA-33 in atherosclerosis etiology and pathophysiology. Atherosclerosis 2013, 227, 201-218, https://doi.org/10.1016/j.atherosclerosis.2012.11.025.

91. Koroleva, I.A.; Nazarenko, M.S.; Kucher, A.N. Role of microRNA in development of instability of atherosclerotic plaques. Biochemistry $\quad$ (Moscow) $\quad 2017, \quad 82, \quad 1380-1390$, https://doi.org/10.1134/S0006297917110165.

92. Heo, K.S.; Fujiwara, K.; Abe, J.I. Shear stress and atherosclerosis. Mol. Cells 2014, 37, 435-440, https://doi.org/10.14348/molcells.2014.0078.

93. He, J.; Luo, Y.; Song, J.; Tan, T.; Zhu, H. Non-coding RNAs and pathological cardiac hypertrophy. In: Noncoding RNAs in cardiovascular diseases. Advances in experimental medicine and biology. Xiao J. (eds) Springer: Singapore. Volume 1229, 2020; pp. 231-245, https://doi.org/10.1007/978-981-15-1671-9_13.

94. Wang, Z.; Zhang, X.J.; Ji, Y.X. Zhang, P.; Deng, K.Q.; Gong, J.; Ren, S.; Wang, X.; Che, I.; Wang, H.; Gao, C. The long non-coding RNA Chaer defines an epigenetic checkpoint in cardiac hypertrophy. Nat. Med. 2016, 22, https://doi.org/10.1038/nm.4179.

95. Liu, C.Y.; Zhang, Y.H.; Li, R.B.; Zhou, L.Y; An, T.; Zhang, R.C,.;Zhai, M.; Huang, Y.;Yan, K.W.; Dong, Y.H.; Ponnusamy, M. LncRNA CAIF inhibits autophagy and attenuates myocardial infarction by blocking p53-mediated myocardin transcription. Nat. Comm. 2018, 9, 1-2, https://doi.org/10.1038/s41467-017-02280y.

96. Kreft, H.; Jetz, W. Global patterns and determinants of vascular plant diversity. Proc. Natl. Acad. Sci. 2007, 104, 5925-5930, https://doi.org/10.1073/pnas.0608361104.

97. Artal-Sanz, M.; Tavernarakis, N. Prohibitin and mitochondrial biology. Trends Endrocrinol. Metab. 2009, 20, 394-401, https://doi.org/10.1016/j.tem.2009.04.004. 
98. Wang, K; Long, B; Zhou, L.Y.; Liu, F.; Zhou, Q.Y; Liu C.Y.; Fan, Y.Y; Li, P.F. CARL IncRNA inhibits anoxia-induced mitochondrial fission and apoptosis in cardiomyocytes by impairing miR-539-dependent PHB2 downregulation. Nat. Comm. 2014, 5, 1-3, https://doi.org/10.1038/ncomms4596.

99. Gao, J.; Chen, X.; Shan, C.; Wang, Y.; Li, P.; Shao, K. Autophagy in cardiovascular diseases: role of noncoding RNAs. Mol. Ther. Nucleic Acids 2020, 23, 101-118, https://doi.org/10.1016/j.omtn.2020.10.039.

100. Saliminejad, K.; Khorram Khorshid, H.R.; Soleymani Fard, S.; Ghaffari, S.H. An overview of microRNAs: biology, functions, therapeutics, and analysis methods. J. Cell. Physiol. 2019, 234, 5451-5465, https://doi.org/10.1002/jcp.27486.

101. Lim, T.B., Lavenni, A., Foo, R.S. Circles in the heart and cardiovascular system. Cardiovasc. Res 2020, 116, 269-278, https://doi.org/10.1093/cvr/cvz227.

102. Luo, B.; Zeng, X.; Liu, P.; Zhu, H. Noncoding RNAs and Heart Failure. Adv. Exp. Med. Biol. 2020, 1229, 215-229, https://doi.org/10.1007/978-981-15-1671-9_12.

103. Zhang, C.; Huo, S.T.; Wu, Z.; Chen, L.; Wen, C.; Chen, H.; Wu, W.D.; Wu, N.; Guan, D.; Lian, S; Yang, B.B. Rapid development of targeting circRNAs in cardiovascular diseases. Mol. Ther. Nucleic Acids 2020, 21, 569-576, https://doi.org/10.1016/j.omtn.2020.06.022.

104. Piccoli, M.T.; Gupta, S.K.; Viereck, J.; Foinquinos, A.; Samolovac, S.; Kramer, F.L.; Garg, A.; Remke, J.; Zimmer, K.; Batkai, S.; Thum, T. Inhibition of the cardiac fibroblast-enriched lncRNA Meg3 prevents cardiac fibrosis and diastolic dysfunction. Circ. Res. 2017, 121, 575-583, https://doi.org/10.1161/CIRCRESAHA.117.310624. 